\title{
Selective attention during scene perception: Evidence from negative priming
}

\author{
ROBERT D. GORDON \\ North Dakota State University, Fargo, North Dakota
}

\begin{abstract}
In two experiments, we examined the role of semantic scene content in guiding attention during scene viewing. In each experiment, performance on a lexical decision task was measured following the brief presentation of a scene. The lexical decision stimulus named an object that was either present or not present in the scene. The results of Experiment 1 revealed no priming from inconsistent objects (whose identities conflicted with the scene in which they appeared), but negative priming from consistent objects. The results of Experiment 2 indicated that negative priming from consistent objects occurs only when inconsistent objects are present in the scenes. Together, the results suggest that observers are likely to attend to inconsistent objects, and that representations of consistent objects are suppressed in the presence of an inconsistent object. Furthermore, the data suggest that inconsistent objects draw attention because they are relatively difficult to identify in an inappropriate context.
\end{abstract}

A typical visual scene contains many objects set against a meaningful background. A goal of an observer viewing such a scene is to identify the scene and the objects contained within it, a process that apparently occurs quite rapidly (see, e.g., Potter, 1976). Because visual attention plays such an important role in perception, understanding how people perceive and represent scenes depends critically on understanding the factors that influence attention during scene viewing. The present set of experiments examines the representation of objects in complex and meaningful scenes, and the role of attention in supporting the development of that representation.

Much recent work on scene representation has been stimulated by demonstrations that observers often fail to notice changes in scene content. Grimes (1996; see also McConkie \& Zola, 1979), for example, demonstrated that observers routinely fail to detect even very large scene changes, as long as those changes are made during a saccade, when vision is suppressed (Volkmann, 1986). Rensink, O'Regan, and Clark (1997) have further shown that a saccade is unnecessary for generating such "change blindness." In their approach, subjects see two alternating versions of a single scene (one original and one changed version). The versions are alternated in rapid succession, separated by a brief blank field; subjects are typically quite poor at detecting the change. Change blindness is

The research reported here was supported by the National Science Foundation under Grant 01322899 and by the National Institutes of Health under Grant 1P20 RR020151. I thank Andrew Hollingworth, Yuhong Jiang, and an anonymous reviewer for their feedback on a previous draft, and Mickey Bahe, Rachel Hanson, Alisha O'Hara, and Travis Roy for their assistance with data collection. Correspondence concerning this article should be addressed to R. D. Gordon, Department of Psychology, North Dakota State University, Fargo, ND 58105 (e-mail: robert.d.gordon@ ndsu.edu). also observed when the change is accompanied by the abrupt appearance of visual stimuli elsewhere in the display (O'Regan, Rensink, \& Clark, 1999) or by an eyeblink (O'Regan, Deubel, Clark, \& Rensink, 2000).

Several accounts of change blindness have emerged that differ with respect to the amount of perceptual scene information represented (see Mitroff, Simons, \& Levin, 2004, for a review). One prominent account attributes change blindness to a sparse and fragile representation of the prechange scene. Rensink and his colleagues (e.g., Rensink, 2000; Rensink et al., 1997), for example, have argued that mental scene representations consist of a small number of objects that are the current focus of attention. Although some representation of those objects may persist following the withdrawal of attention, Rensink argues that what survives is a representation of object type, rather than a perceptual representation. By this account, failure to detect a change reflects either a failure to encode the changed object (because it is not the focus of attention), or a failure to retain the representation across the change. Irwin and his colleagues (e.g., Irwin \& Andrews, 1996; Irwin \& Gordon, 1998; Irwin \& Zelinsky, 2002) have similarly argued that memory across a saccade consists of a small set of object token representations in visual shortterm memory (VSTM), combined with some residual activation in a semantic network. The set of objects represented are those objects that have recently been attended.

A second class of explanations posits that rather than (or in addition to) change blindness reflecting a sparse or fragile representation, it may reflect a failure to compare pre- and postchange visual information. That is, even if a richly detailed representation of the scene were maintained, change detection may still fail if that representation is not compared with the postchange scene (see, e.g., Hollingworth, 2003; Mitroff et al., 2004). Support for this explanation comes from a growing body of evidence sug- 
gesting that scene representations contain more information than was previously thought.

Hollingworth (2004, 2005; Hollingworth \& Henderson, 2002), for example, has demonstrated that memory for the visual properties of objects in scenes may be retained for much longer than the presumed duration of VSTM. In one experiment, subjects viewed a set of scenes, and then, $24 \mathrm{~h}$ later, a set of test scenes. Some of the test scenes were identical to the original scenes, whereas in others, one of the objects was either replaced with a different object token, or was rotated from its original position. The subjects' task was to indicate whether the target object had changed. Despite the 24-h delay, subjects were significantly above chance on the change-detection task. Hollingworth (2005) argued that the perceptual properties of viewed objects may be represented in a visual long-term memory (VLTM), in addition to VSTM.

It is important to note that Hollingworth does not argue that durable representations of objects in scenes are constructed in parallel across the scene during initial scene viewing. Instead, each object representation is formed following attention to that object. Although the initial scene representation may be relatively sparse (as Rensink, 2000, argues), a more complex and detailed scene representation may develop across several fixations.

This stands in marked contrast to the limited scene representation proposed by Rensink (2000) and others. What both conceptualizations of scene representation have in common, however, is that they assign a central role to selective attention. Under Rensink's explanation, representation is limited to currently attended objects. Similarly, according to Irwin and Andrews (1996), representation of precise perceptual information is limited to VSTM, access to which is mediated by attention. Finally, Hollingworth (2005) posits an enduring representation of a relatively large number of scene objects, but only those objects that have been fixated or attended.

Because the most prominent accounts of scene representation assign a central role to visual attention, fully understanding how scenes are perceived and represented requires understanding the factors that guide attention during scene viewing. It is those factors that are the focus of the present investigation. In particular, the present research focuses on the role of semantic factors in guiding attention during the initial fixation on a scene.

\section{Attention During Scene Perception}

There is considerable disagreement concerning the extent to which attention is guided by a scene's semantic content. Most studies that have investigated the issue have done so by measuring subjects' eye movements while they view scenes (e.g., De Graef, Christiaens, \& d'Ydewalle, 1990; De Graef, De Troy, \& d'Ydewalle, 1992; Henderson, Weeks, \& Hollingworth, 1999; Loftus \& Mackworth, 1978). Loftus and Mackworth, for example, had subjects view scenes containing a semantically consistent or inconsistent object. Semantically consistent objects were those with a high a priori probability of occurring within the scene (e.g., an octopus in an ocean scene). Semanti- cally inconsistent objects, in contrast, had a low a priori probability of occurring within the scene (e.g., a tractor in an ocean scene). Loftus and Mackworth reported that subjects tended to fixate semantically inconsistent objects earlier than consistent objects during scene viewing. They speculated that the conflict between inconsistent object identity and scene context is likely to draw attention, increasing the probability of fixation. De Graef et al. (1992) have also suggested that attention may be guided by semantic factors during scene viewing.

Others have suggested, however, that attention is guided by perceptual factors alone, and not by a scene's semantic content. Henderson et al. (1999), for example, using a procedure very similar to that used by Loftus and Mackworth (1978), failed to find an advantage for inconsistent objects. Thus, the results of eyetracking studies examining guidance of attention by semantic factors have been equivocal.

Gordon (2004), using a probe-discrimination task, examined the guidance of attention by scene semantic content within a single fixation. Subjects viewed scenes that contained a target object that was either semantically consistent or inconsistent with the scene context. In one experiment, each scene was presented for either 53 or $147 \mathrm{msec}$. The scene was then replaced by a pattern mask; embedded in the mask was a probe target ( $\%$ or \&) that the subject was required to identify. The probe location matched the screen location of the consistent or inconsistent target object in the scene. Because probe identification is affected by attention, performance on the probe task serves as an index of attention during scene viewing: Subjects should be faster when the probe appears at an attended location than when it appears at an unattended location.

When the scene was presented for just $53 \mathrm{msec}$, probe reaction time (RT) did not differ for consistent- and inconsistent-object probes. However, when the scene was presented for $147 \mathrm{msec}$, subjects were faster to identify inconsistent-object probes than consistent-object probes, suggesting that they were likely to attend to inconsistent objects within approximately $150 \mathrm{msec}$ of scene onset. This was confirmed in subsequent experiments, including one in which the inconsistent object was never actually probed. In that experiment, scenes contained either one inconsistent and one consistent object, or two consistent objects, and the probed object was always consistent with the scene context. Gordon (2004) found that the presence of an inconsistent object elsewhere in the scene was associated with an RT cost to consistent-object probes.

One interpretation of these results (and the interpretation favored by Gordon, 2004) is that attention was drawn to the location of the inconsistent object because of the semantic conflict between that object and the scene context. Under this semantic conflict interpretation, attention is drawn to such objects in order to resolve the conflict, or because objects that are not redundant with their context carry more information than other objects (see, e.g., Loftus \& Mackworth, 1978). An alternative interpretation is that attention is drawn to areas containing objects that are particularly difficult to identify (see, e.g., Johnston, Hawley, Plewe, Elliott, \& DeWitt, 1990). Under this local 
processing difficulty interpretation, some objects will be systematically more difficult to identify than others, and will therefore represent an area of perceptual dysfluency that draws attention. If, as some have argued (e.g., Davenport \& Potter, 2004), object recognition is influenced by contextual information, inconsistent objects (whose identification is not supported by the scene context) will be more likely to constitute such areas than will consistent objects. Thus, attention may be drawn to such objects, not because their identities conflict with the scene context, but because they are difficult to identify at all in an inappropriate context.

One way to resolve this issue is to look for evidence that inconsistent objects are identified early in scene perception; although the semantic conflict account assumes that inconsistent objects are often identified, the local processing difficulty account assumes that they are not. Gordon (2004) addressed this by asking subjects to identify the probed object on every trial. Using the same scenes as in the present experiments, Gordon found that subjects were able to identify the inconsistent object on only $15 \%$ of trials. (In contrast, consistent objects were correctly identified on $40 \%$ of trials.) Such poor performance, although inconsistent with the semantic conflict account, appears to be supportive of the local processing difficulty account.

The approach used by Gordon (2004), however, represents an imperfect test of object processing, for at least two reasons. First, the task required subjects to explicitly identify the probed object by naming it; such a task may not be sensitive to many processes associated with object identification. Second, the identification task was secondary to the probe identification task, and always followed the probe identification response; object representations that were activated during scene viewing may have decayed while the probe identification task was carried out.

In the present study, I use a more sensitive measure of object processing that overcomes the shortcomings of the explicit recognition task used by Gordon (2004). In two experiments, I examine semantic priming from consistent and inconsistent objects using a lexical decision task. By revealing the processing of consistent and inconsistent objects, these experiments provide a strong test of the semantic conflict and local processing difficulty accounts of Gordon's results. This approach also may provide converging evidence for selection of inconsistent objects during scene perception, as described below.

\section{Priming and Attention}

A considerable body of evidence shows that responses to a target stimulus may be influenced by prior exposure to the same or a related stimulus (see Neely, 1991, for a review). This priming of the response takes two forms. Positive priming occurs when the response to the target stimulus is facilitated by a previous or concurrent event. For example, subjects may decide more quickly that NURSE is a word in a context in which they have seen the word NURSE or DOCTOR at another location or on a previous trial, than in the context of having seen an unrelated word (see, e.g., Schvaneveldt \& Meyer, 1973). It has been shown that attention to a stimulus generally produces positive priming when that stimulus (or a related stimulus) is subsequently encountered (see, e.g., Ortells \& Tudela, 1996). In the present experiment, positive priming is expected from inconsistent objects, if such objects are attended and identified. If, however, inconsistent objects are not identified (as predicted by the local processing difficulty account), they should not prime a subsequent response.

Negative priming occurs when a prior event interferes with a response to a target stimulus (see, e.g., Ortells \& Tudela, 1996; Tipper, 1985; Tipper \& Cranston, 1985; Tipper \& Driver, 1988). Tipper (1985), for example, showed subjects two overlapping objects on every trial. One object was drawn in red and the other in green, and a subject's task on every trial might be to name the picture drawn in red. In the critical condition, the object that was ignored on one trial became the object that was to be named on the following trial. In that condition, the subjects named the target more slowly than if it was preceded by a neutral object in the previous trial. Tipper attributed this result to selective attention (see also Fox, 1995). On each trial, he argued, the subjects attended to one object and ignored the other. Selection of the target stimulus was accompanied by inhibition of the distractor, which carried over to the following trial.

This inhibition appears to follow semantic processing of unattended stimuli, and is not specific to visual form. Tipper and Driver (1988), for example, measured negative priming using a procedure that was quite similar to that used by Tipper (1985), except that the prime and target stimuli did not always have the same form. If, on one trial, the attended stimulus was a picture of a hammer and the ignored stimulus was a picture of a dog, the target on the next trial might be the word DOG (in the ignored repetition condition), or CAT (in the ignored semantic condition), or FOOT (in the control condition). The subjects' task was to name the target on each trial. Despite the fact that the target was never physically identical to the prime, negative priming was observed for both the repetition and semantic conditions; that is, the subjects named the targets more slowly in the ignored repetition and ignored semantic conditions than in the control condition. Tipper and Driver concluded that semantic processing occurs even for unattended stimuli, and that inhibition acts on postcategorical representations during selection.

Priming experiments thus may reveal two aspects of scene processing simultaneously. First, they may reveal evidence of conceptual processing of scene content. Second, they reflect attentional selection that occurs during scene processing. Stimuli that are attended and identified during scene viewing are likely to produce positive priming on a subsequent task. Stimuli that are ignored, in contrast, may be expected to produce negative priming. In the present set of experiments, I examine priming from consistent and inconsistent objects presented within scenes, in order to measure the conceptual processing and attentional selection of those objects.

VanRullen and Koch (2003) present data that suggest that objects embedded in complex scenes may produce 
negative priming. In their experiment, subjects viewed a series of 10 scenes, each of which contained 10 discrete objects. Each scene was presented for $250 \mathrm{msec}$ and then masked. Following scene presentation, a list of 20 object names was presented for a recall task; the list included the 10 objects present in the scene, along with 10 plausible (but absent) objects. The subjects indicated which of the objects was present in the scene they had just viewed. Shortly afterward, the subjects performed a word-picture matching task, in which an object name was followed by an object image, and the subject indicated whether or not the name matched the image. The objects presented included the 10 objects that had been embedded in the scene they had viewed, along with several novel objects that had not been present in the scene. RTs on this task were compared with those of a control group that did not view the scenes before performing the task.

VanRullen and Koch (2003) examined RTs for the objects that had been reported during the recall task, and for the objects that had been missed. The results revealed a small positive priming effect. Though the finding was not significant, RTs tended to be shorter for subjects who reported seeing the target object in the scene than for control subjects. Importantly, there was also a significant negative priming effect; RTs were longer for subjects who did not report the target object's presence in the scene than for control subjects. Furthermore, the RTs to missed objects were also longer than the same subjects' RTs to the novel objects. VanRullen and Koch, consistent with Tipper and Driver (1988), suggest that objects within a scene compete for attention. Those objects that are selected receive enhanced processing, whereas those that are not selected are suppressed during selection. Because certain objects were not selected, subjects fail to report their presence when asked to recall the scene content. Nonetheless, evidence of their suppression is observed as negative priming on a subsequent task.

\section{The Present Experiments}

Gordon (2004) reported that attention may be guided within a single fixation by the semantic content of a scene. In those experiments, data from a probe discrimination task suggested that subjects allocate attention quite rapidly to the location of semantically inconsistent objects. In the present set of experiments, I seek converging evidence for the semantic guidance of attention, using a new approach: the measurement of positive and negative priming from scene content.

This evidence will provide a test of two competing explanations for Gordon's (2004) results. One explanation is that the semantic properties of the inconsistent object draw attention. According to this explanation, subjects attend to the inconsistent object because they have at least partially identified the object, and this identification produces a conflict with the developing scene representation; attention is drawn to the source of the conflict. This is the interpretation offered by Gordon, and, because it presupposes inconsistent object identification, it predicts positive priming from the inconsistent object in the scene. Another possibility, however, is that attention was drawn to the inconsistent object in Gordon's experiments because the object could not be identified. According to this account, identification of the consistent objects within the scene is enhanced by scene context (e.g., Davenport \& Potter, 2004). The inconsistent object, on the other hand, is relatively difficult to identify, because its identification is not supported by the scene context. This area of local processing difficulty may thus constitute a region of perceptual dysfluency to which attention is drawn (Johnston et al., 1990). This account predicts no positive priming from the inconsistent object, because it assumes that the inconsistent object is not fully identified during scene viewing. Thus, the presence or absence of positive priming of inconsistent object identity will permit a test of these two competing hypotheses. Regardless of whether or not positive priming is observed, however, negative priming from consistent object identity is expected to follow selection of the inconsistent object in the scene. Such a result would suggest that attention during scene viewing is guided by the outcome of semantic scene and object processing.

\section{EXPERIMENT 1}

The purpose of Experiment 1 was to examine attentional selection during the perception of meaningful scenes, and to examine the processing of consistent and inconsistent objects embedded in those scenes. On each trial, a scene was presented for $100 \mathrm{msec}$, and then masked. A target letter string was then presented, and the subjects' task was to indicate whether or not it formed a familiar English word (i.e., subjects performed a lexical decision task). Four types of target words were used: consistent-present targets named consistent objects present in the scene; inconsistentpresent targets named inconsistent objects present in the scene; consistent-absent targets named consistent objects not present in the scene; and inconsistent-absent targets named inconsistent objects not present in the scene. Priming was assessed by comparing RTs to present targets to RTs to absent targets.

\section{Method}

Subjects. Twenty-eight undergraduate students at North Dakota State University participated in the experiment in exchange for course credit. All of the subjects had normal or corrected-to-normal vision.

Stimuli. The stimuli consisted of a set of 96 scenes (four versions of 24 scenes) derived from a set of base scenes and objects developed by Hollingworth and Henderson (1998) and De Graef et al. (1990). Most of the scenes were tracings of photographs of real-world scenes. In each scene used in the "word" trials of Experiment 1, a consistent object and an inconsistent object were inserted into the scene. The location of the critical objects varied across scene versions. In most cases, the inserted objects were the objects previously used by Gordon (2004). In addition to the 48 scenes used in the word trials, the set of 24 base scenes was modified to create a separate set of 48 scenes for use in the nonword trials. As in the word trials, the scenes always contained inserted consistent and inconsistent objects, and the locations of these objects varied and were counterbalanced across scene versions. Figure 1 depicts a typical scene used in Experiment 1. 
The critical inserted objects used in the experiment were created independently from the scenes; as Henderson et al. (1999) have suggested, this approach reduces (but does not eliminate) the possibility that semantically inconsistent objects will be perceptually salient, relative to semantically consistent objects. At a viewing distance of $57 \mathrm{~cm}$, the scenes subtended a visual angle of $22.3^{\circ}$ horizontally and $13.6^{\circ}$ vertically. All of the scenes were followed by a blackand-white pattern mask, which consisted of jumbled lines, arcs, and angles; the size of the pattern mask matched that of the scenes. The size of the critical objects varied. The critical consistent objects had a mean size of $2.1^{\circ}\left(S D=0.6^{\circ}\right)$ horizontally and $2.1^{\circ}\left(S D=0.6^{\circ}\right)$ vertically, whereas the critical inconsistent objects had a mean size of $2.2^{\circ}\left(S D=0.6^{\circ}\right)$ horizontally and $2.2^{\circ}\left(S D=0.7^{\circ}\right)$ vertically.

Each critical object was inserted in two locations across scene versions, in order to reduce the predictability of the object location within each scene (that is, in order to reduce the number of times that a critical object appeared within a particular screen location). The mean eccentricity was $4.5^{\circ}$ (range $=0.8^{\circ}-8.8^{\circ} ; S D=2.2^{\circ}$ ). Because the stimuli were created from scenes used by Gordon (2004), the eccentricities matched the eccentricities in those experiments. Given Gordon's results, it is clear that objects at these eccentricities are capable of attracting attention on the basis of their semantic relationship with the scene context.

In addition to the scenes, 96 words and 48 nonwords were used as targets. Half of the word stimuli named objects present in the scenes and half named objects not present in the scenes. The present-object names had a mean length of 6.1 letters and a mean written frequency of 42.9 times per million (Francis \& Kučera, 1982); the absent-object names had a mean length of 6.7 letters and a mean written frequency of 49.6 times per million. The nonword stimuli were taken from Sereno (1991), had a mean length of 5.6 letters, and followed standard English rules of orthography and pronunciation.

Apparatus. The stimuli were presented at a screen resolution of $1,024 \times 768$ pixels on an Envision Peripherals EN-710E color monitor; the monitor was configured to have a refresh rate of $60 \mathrm{~Hz}$. Stimulus presentation was controlled with a 2.4-GHz Pentium 4 computer (Technology Distribution Network) running E-Prime experiment control software (Psychology Software Tools, Inc.). The scene contours were displayed in gray scale, and noncontour scene areas were drawn in white. The subject responses on the lexical decision task were collected by sampling at $1000 \mathrm{~Hz}$ from a response box (Psychology Software Tools, Inc.) interfaced through the computer's serial port.

Procedure. The subjects began each trial by fixating a central fixation cross and pressing one of the two response keys. The fixation cross remained visible for $1 \mathrm{sec}$, and was then replaced with a blank white screen for $500 \mathrm{msec}$. The scene was then presented for $100 \mathrm{msec}$, followed immediately by a pattern mask presented for $100 \mathrm{msec}$. Following the mask, a blank white screen was presented for $100 \mathrm{msec}$ and then the target letter string was presented. Thus, the scene-target stimulus onset asynchrony (SOA) was $300 \mathrm{msec}$ on every trial. Vanderwart (1984) has found substantial priming of lexical decision by line drawings of isolated objects at this SOA.

The subjects were instructed to indicate, as quickly as possible, whether or not the letter string formed a familiar English word. The target remained visible until the subject pressed one of two keys to indicate his or her response. All of the subjects pressed the left response key when the target string was a word, and pressed the right response key when the target string was not a word. On half of the trials, the target stimulus was a nonword. On the remaining trials, one of four word types (consistent-present, inconsistent-present, consistent-absent, and inconsistent-absent) was presented. As explained previously, these four target types permitted an assessment of priming from inconsistent- and consistent-object identity.

Before beginning the block of experimental trials, the subjects completed one practice block of 12 trials. The procedure on each practice trial matched that of the experimental trials. On half the trials, the target was a word, and on the other half, the target was a nonword. Of the word targets, half were consistent with the scene and half were not. Following the practice block, the subjects completed one block of 96 experimental trials. The subjects required approximately 15 min to complete the block of experimental trials. Of the 96 experimental trials, 48 were nonword trials, whereas the remaining 48 were trials in which the target was a word. Those trials were equally divided among the four target types (consistentpresent, consistent-absent, inconsistent-present, and inconsistentabsent). Thus, the experiment had a 2 (word vs. nonword) $\times 2$ (target consistency) $\times 2$ (target presence) design. However, analyses were conducted using data from the word trials only.

It is important to establish that RT differences observed between the present and absent targets reflect effects of scene content, rather than intrinsic differences among the stimuli. To test this, 48 subjects

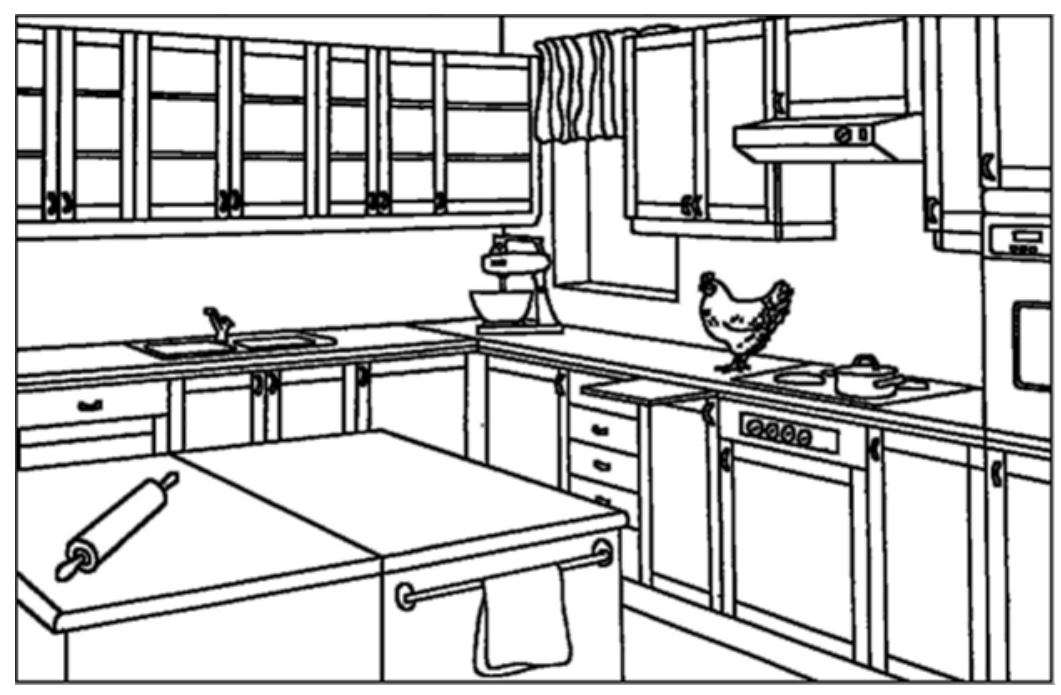

Figure 1. Example of a scene used in Experiment 1. The consistent target object in the scene is the mixer; the inconsistent target object is the chicken. 
participated in an initial study in which the word targets were preceded by scenes that did not contain the critical objects (e.g., the kitchen scene was presented without a mixer or a chicken inserted). In all other respects, the experimental procedure was identical to that used in Experiment 1. The results revealed no significant RT differences between consistent-present words $(M=581 \mathrm{msec})$ and consistent-absent words $(M=578 \mathrm{msec})$, or between inconsistentpresent $(M=586 \mathrm{msec})$ and inconsistent-absent $(M=593 \mathrm{msec})$ words (both $t \mathrm{~s}<1$ ). Thus, it appears unlikely that intrinsic stimulus differences can account for any priming effects observed in Experiments 1 and 2 .

\section{Results}

Before lexical decision RTs were analyzed, the data were trimmed by eliminating RTs greater than $2 \mathrm{sec}$, or those that differed by more than 2.5 standard deviations from that subject's mean RT for that condition (consistent/ inconsistent and presence/absence). This criterion eliminated $2.6 \%$ of the trials from analysis.

Mean RTs and error rates for each condition are reported in Table 1. The results of an ANOVA performed on the RTs revealed a main effect of target consistency, with the subjects responding faster to targets naming consistent objects $(M=564 \mathrm{msec})$ than to targets naming inconsistent objects $(M=592 \mathrm{msec})\left[F(1,27)=13.9, M S_{\mathrm{e}}=1,517, p<.01\right]$. There was no main effect of target presence $[F(1,27)=1.5$, $\left.M S_{\mathrm{e}}=1,305, p>.05\right]$. Furthermore, there was no significant interaction between target consistency and target presence $\left[F(1,27)=1.9, M S_{\mathrm{e}}=2,462, p>.05\right]$. Nonetheless, planned comparisons were conducted to assess priming from consistent and inconsistent objects in the scenes.

The first planned comparison assessed priming from the identity of the inconsistent object, and was measured by comparing the inconsistent-absent condition with the inconsistent-present condition. Inconsistent objects did not significantly prime the lexical decision response: The subjects responded as quickly to inconsistent-present targets $(M=595 \mathrm{msec})$ as to inconsistent-absent targets $(M=$ $588 \mathrm{msec})[t(27)<1]$. Thus, these data do not suggest that the subjects identified the inconsistent object present in the scene during scene viewing.

The second planned comparison assessed priming from the identity of the consistent object by comparing the consistent-absent condition with the consistent-present condition. The planned comparison revealed significant negative priming from the consistent-present object $[t(27)=2.407, p<.05]$; the subjects responded faster to a consistent-absent target $(M=552 \mathrm{msec})$ than to a consistent-present target $(M=576 \mathrm{msec})$. In addition to

Table 1

Lexical Decision Times (RTs, in Milliseconds) and Error Rates in Experiment 1

\begin{tabular}{|c|c|c|c|c|}
\hline \multirow{3}{*}{$\begin{array}{c}\text { Target } \\
\text { Presence }\end{array}$} & \multicolumn{4}{|c|}{ Target Consistency } \\
\hline & \multicolumn{2}{|c|}{ Consistent } & \multicolumn{2}{|c|}{ Inconsistent } \\
\hline & RT & PE & RT & $\mathrm{PE}$ \\
\hline Present & 576 & 2.9 & 588 & 3.3 \\
\hline Absent & 552 & 2.0 & 595 & 5.8 \\
\hline Priming effect & -24 & -0.9 & 7 & 2.5 \\
\hline
\end{tabular}

confirming consistent-object processing, this result also suggests that consistent-object representations were suppressed prior to the response. Implications of the planned comparisons are discussed further below.

The results of an ANOVA performed on error rates were generally consistent with the RT analyses. There was a main effect of consistency, with the subjects making fewer errors for consistent targets $(M=2.5 \%)$ than for inconsistent targets $(M=4.6 \%)\left[F(1,27)=4.5, M S_{\mathrm{e}}=\right.$ $26, p<.05]$. There was no effect of presence $[F(1,27)=$ $\left.1.2, M S_{\mathrm{e}}=17, p>.05\right]$. There was also no significant interaction between consistency and presence $[F(1,27)=$ $\left.2.8, M S_{\mathrm{e}}=30, p>.05\right]$. As in the RT analyses, planned comparisons were conducted to assess the two sources of priming. This was done by making the same comparisons outlined in the description of the RT analyses. These comparisons revealed no significant priming from the inconsistent object present in the scene $[t(27)=2.006, p>$ $.05]$ or from the consistent object in the scene $[t(27)<1]$. Nonetheless, subjects tended to make fewer errors to consistent-absent targets $(M=2.0 \%)$ than to consistentpresent targets $(M=2.9 \%)$. This trend is consistent with the negative priming effect observed in the RT analyses.

\section{Discussion}

Gordon (2004) reported that subjects are more likely to attend to inconsistent than to consistent objects within scenes. The results of Experiment 1 provide converging evidence for this claim. Consistent objects presented within scenes containing inconsistent objects produced negative priming on a subsequent lexical decision task, suggesting that consistent object representations were suppressed during the viewing of those scenes. According to a prominent view of attentional dynamics (see, e.g., Tipper \& Driver, 1988), such suppression is an expected consequence of the selection of an object from a set of distracting objects. The present results suggest that this general principle applies to objects embedded within complex, meaningful scenes; under such conditions, the selection of an inconsistent object is accompanied by the suppression of other objects within the scene.

Although Gordon (2004) presented scenes for $150 \mathrm{msec}$, the present experiment used scene durations of just $100 \mathrm{msec}$. It is tempting to conclude, therefore, that the results of Experiment 1 suggest that inconsistent object selection emerges even earlier during scene viewing than Gordon claimed. However, the present experiments are not well-suited to assessing the temporal dynamics of semantic processing and shifts of attention during scene viewing. Gordon assessed selection of the inconsistent object immediately following scene offset. In contrast, in the present experiment, processing of the scene, and the objects within it, was not assessed until the target word appeared, $200 \mathrm{msec}$ following scene offset. Because considerable conceptual processing is known to occur during this interval (see, e.g., Potter, 1976), even when the scene is followed by a pattern mask (Loftus, Hanna, \& Lester, 1988), at least some of the processing that produces nega- 
tive priming on the lexical decision task may follow the offset of the scene itself.

In addition to providing additional evidence that attention is likely to select inconsistent objects during scene viewing, Experiment 1 also provided a test of two competing accounts of that phenomenon. According to the semantic conflict account favored by Gordon (2004), attention is drawn to the location of a semantic conflict between the scene context and the identity of the inconsistent object. Crucially, this explanation assumes that the inconsistent object is identified rapidly, and that it is this identification that leads to attentional selection. In contrast, the local processing difficulty account assumes that identification of an inconsistent object is impaired by a lack of contextual support. According to this account, this identification difficulty produces a perceptual trouble spot that draws attention. According to this explanation, therefore, the identification of inconsistent objects should be quite poor.

The results of Experiment 1 support the latter account, and suggest the following account of early scene processing. When a scene is encountered, observers begin to identify the gist of the scene and the identities of objects within the scene. Gist identification occurs very rapidly (see, e.g., Potter, 1976), and knowledge of the scene's gist may therefore enhance the perception of objects within the scene (see, e.g., Davenport \& Potter, 2004). This enhancement will only occur, however, for those objects with a high a priori probability of occurring in the scene; that is, enhancement is expected for consistent objects, but not for inconsistent objects. In the absence of contextual support, the briefly presented inconsistent objects will be more difficult to identify than will the consistent objects (Davenport \& Potter, 2004), and will thus constitute a perceptual trouble spot. As Johnston and his colleagues have demonstrated (see, e.g., Johnston et al., 1990), such areas of local processing difficulty are likely to draw attention. This is accompanied by the suppression of other objects in the scene (see, e.g., Neill, 1977; Tipper, 2001; Tipper, Brehaut, \& Driver, 1990; VanRullen \& Koch, 2003), and this suppression carries consequences for later object processing. The fact that the suppression produces negative priming on a lexical decision task suggests, consistent with Tipper and Driver (1988), that the suppression operates on postcategorical object representations.

The clear implication of this explanation for the result of Experiment 1 is that the presence of an inconsistent object in the scene should be necessary in order to obtain negative priming by the consistent scene object. This was tested in Experiment 2.

\section{EXPERIMENT 2}

In Experiment 2, the presence of an inconsistent object in the scene was manipulated between subjects. In the inconsistent-distractor condition, every scene contained both an inserted consistent and inconsistent object, as in Experiment 1 . In the consistent-distractor condition, however, the stimuli were modified so that the scenes contained no inconsistent objects. Instead, scenes in this condition contained two inserted consistent objects.

According to the attentional selection account of the results of Experiment 1, negative priming from consistent objects reflects attention to inconsistent objects; selection of an inconsistent object is accompanied by suppression of other (consistent) objects in the scene. This suppression produces negative priming on the subsequent lexical decision task. If the attentional selection account is correct, then there should be no negative priming from consistent object identity in the consistent-distractor condition, in which no inconsistent object is present to reliably draw attention. In contrast, negative priming should occur in the inconsistent-distractor condition, replicating the results of Experiment 1.

\section{Method}

Subjects. Forty-eight undergraduate students from North Dakota State University participated in exchange for course credit. All of the subjects had normal or corrected-to-normal vision, and none had participated in Experiment 1.

Stimuli. For trials in the inconsistent-distractor condition, the stimuli consisted of 24 base scenes that were identical to the scenes used in Experiment 1. For trials in the consistent-distractor condition, the stimuli consisted of scenes that were identical to those used in the inconsistent-distractor condition, except that each scene contained two inserted consistent objects. The consistent target was presented in the same location as the consistent target in the inconsistent-distractor condition, whereas the consistent distractor was presented at the same location as the inconsistent distractor in that condition. Thus, although each scene contained two inserted objects, only in the inconsistent-distractor condition was one of the inserted objects inconsistent with the scene context. The set of scenes used was previously used by Gordon (2004) to examine attentional allocation within scenes. Viewing conditions were the same as in Experiment 1, as were the target words and nonwords.

Apparatus. The apparatus was the same as that used in Experiment 1 .

Procedure. The procedure was similar to that used in Experiment 1 . The subjects completed 12 practice trials followed by one block of 192 experimental trials. Half of the experimental trials were nonword trials, which were not analyzed. Of the 96 word trials, 48 of the targets named consistent objects and 48 named inconsistent objects. Of those, half named objects that were present in the scenes and half named objects that were not present. Furthermore, on half of the consistent object trials, an inconsistent distractor was present, whereas on the other half of the trials, the distractor was another consistent object. On inconsistent object trials, an inconsistent object was always present in the scene.

\section{Results}

Before analyses were conducted, the data were trimmed by eliminating trials with RTs greater than $2 \mathrm{sec}$, or those that differed by more than 2.5 standard deviations from that subject's mean RT for that condition (consistent/ inconsistent and presence/absence). This criterion eliminated $3.3 \%$ of the trials from analysis.

Mean RTs and error rates for each condition are reported in Table 2. Because the experiment did not use a fully factorial design, two separate analyses were conducted; one analysis focused exclusively on the consistent object trials, whereas the other focused on the inconsistent object trials alone. 
Table 2

Lexical Decision Times (RTs, in Milliseconds) and Error Rates in Experiment 2

\begin{tabular}{|c|c|c|c|c|}
\hline \multirow{3}{*}{$\begin{array}{c}\text { Target } \\
\text { Presence }\end{array}$} & \multicolumn{4}{|c|}{ Target Consistency } \\
\hline & \multicolumn{2}{|c|}{ Consistent } & \multicolumn{2}{|c|}{ Inconsistent } \\
\hline & RT & $\mathrm{PE}$ & RT & $\overline{\mathrm{PE}}$ \\
\hline \multicolumn{5}{|c|}{ Inconsistent Distractor } \\
\hline Present & 594 & 4.2 & 576 & 2.5 \\
\hline Absent & 572 & 3.4 & 578 & 4.8 \\
\hline Priming effect & -22 & -0.8 & 2 & 2.3 \\
\hline \multicolumn{5}{|c|}{ Consistent Distractor } \\
\hline Present & 565 & 1.9 & - & \\
\hline Absent & 564 & 2.1 & - & \\
\hline Priming effect & -1 & 0.2 & - & \\
\hline
\end{tabular}

The results of an ANOVA performed on RTs for the consistent object trials revealed a marginal effect of distractor type, because subjects tended to respond more slowly when the distractor was an inconsistent object $(M=583 \mathrm{msec})$ than when it was another consistent object $(M=565 \mathrm{msec})\left[F(1,47)=3.8, M S_{\mathrm{e}}=4,237, p=\right.$ .056]. There was no main effect of target presence $(F<$ $1)$, and the interaction of target presence and distractor type failed to reach significance $\left[F(1,47)=1.9, M S_{\mathrm{e}}=\right.$ $2,815, p>.05]$.

Nevertheless, planned comparisons were conducted to test the experimental hypotheses. The results for trials in which the distractor was an inconsistent object replicated the results of Experiment 1. That is, the subjects responded more quickly to consistent-absent targets $(M=572 \mathrm{msec})$ than to consistent-present targets $(M=594 \mathrm{msec})$ when there was an inconsistent distractor present in the scene $[t(47)=2.083, p<.05]$. This negative priming was not obtained, however, when the distractor was another consistent object. In that condition, the subjects responded equally quickly to consistent-present $(M=565 \mathrm{msec})$ and consistent-absent $(M=564 \mathrm{msec})$ targets $[t(47)<1]$. Thus, the results support the central experimental hypothesis: Negative priming was observed when an inconsistent distractor was present, but not when it was absent.

An additional analysis examined the effect of target presence for the inconsistent-object trials. As in Experiment 1, the subjects responded equally quickly in the inconsistentpresent $(M=576 \mathrm{msec})$ and inconsistent-absent $(M=$ $578 \mathrm{msec}$ ) conditions. Thus, as in Experiment 1, there is no evidence for semantic processing of the inconsistent object in the scene.

The results of an ANOVA performed on the error rates for the consistent object trials revealed a significant main effect of distractor type $\left[F(1,47)=5.5, M S_{\mathrm{e}}=28, p<\right.$ $.05]$, with the subjects making more errors when the distractor was an inconsistent object $(M=3.8 \%)$ than when it was a consistent object $(M=2.0 \%)$. There was no effect of target presence, and no interaction between distractor type and target presence (both $F \mathrm{~s}<1$ ). Analyses performed on the inconsistent-target trials revealed that the subjects made more errors to inconsistent-absent targets
$(M=4.8 \%)$ than to inconsistent-present targets $(M=$ $2.5 \%)[t(47)=3.009, p<.05]$.

As with the RT data, planned comparisons were conducted to test experimental hypotheses. There was no difference in the number of errors made to consistent-present and consistent-absent targets, in either the inconsistent- or the consistent-distractor conditions (both $t \mathrm{~s}<1$ ). Thus, the error analyses reveal no speed-accuracy trade-off that might account for the negative priming observed in the RT data.

\section{Discussion}

The results of Experiment 2 are consistent with an attention-based account, as offered following Experiment 1 . When an inconsistent object was present in the scene, it was likely to be the focus of attention (Gordon, 2004). As a consequence, representations of consistent scene objects were likely to be suppressed during scene viewing, leading to negative priming from those objects. This negative priming was observed in Experiment 1, and was replicated in Experiment 2. In the absence of an inconsistent object (or another perceptually or conceptually salient object), however, reliable suppression of the consistent object did not occur, and no negative priming was observed.

\section{GENERAL DISCUSSION}

The goal of the present set of experiments was to examine the guidance of visual attention by a scene's semantic content, and to examine the consequence of attentional selection on the representation of objects within scenes. The results of Experiment 1 revealed no significant positive priming from inconsistent objects embedded in scenes, but significant negative priming from consistent objects within the same scenes. The results of Experiment 2 confirmed that consistent objects produce negative priming only in the presence of an inconsistent object elsewhere in the scene. These experiments, in conjunction with the results of Gordon (2004), provide converging evidence that attention is guided by semantic factors early in scene viewing. Gordon, using a spatial probe technique, argued that observers are likely to attend very quickly to objects whose identities conflict with the context in which they appear. The present experiments confirm Gordon's conclusions, and support a local processing difficulty account of the selection of inconsistent objects. Furthermore, the results reveal the consequences of attentional selection for the representation of objects contained within a scene.

\section{Attentional Dynamics During Scene Viewing}

When a scene is first encountered, information about the general scene category (e.g., bedroom, kitchen, etc.) is acquired very rapidly (see, e.g., Potter, 1976). As Schyns and Oliva (1994) have argued, scene categorization need not involve prior identification of objects within the scene. Indeed, they found that subjects were able to discriminate among scene categories on the basis of low spatial frequency alone, despite the fact that the low spa- 
tial frequency information did not support identification of objects within the scene. The configuration of scene elements thus appears to be sufficient to support scene categorization (see, e.g., Biederman, 1990), greatly increasing its efficiency.

Though scene categorization may proceed in the absence of local object information, this does not imply that there is no interaction between scene categorization and object identification. It is possible, for example, that perception of objects within the scene is influenced by the scene context. This is a somewhat controversial claim. The results of a number of studies have appeared to show that scene context influenced object perception (e.g., Biederman, Mezzanotte, \& Rabinowitz, 1982; Boyce \& Pollatsek, 1992). Hollingworth and Henderson (1998, 1999), however, have argued that the results of those studies reflect response bias, and have suggested instead that object identification is functionally isolated from contextual information. More recently, however, Davenport and Potter (2004) have presented evidence for contextual effects on object identification, in a series of experiments designed to rule out a bias-based account. In one experiment, subjects viewed color photographs consisting of a large foreground object superimposed on a background scene; the foreground object was either consistent or inconsistent with the scene context. Each scene was presented for $80 \mathrm{msec}$, and was then masked. The subject's task was to type in the name of the foreground object immediately following the offset of the mask. Davenport and Potter found that the subjects correctly identified consistent objects more often than inconsistent objects, suggesting that the scene context facilitated the perception of the consistent objects. Interestingly, Davenport and Potter also found that the identification of the scene context itself was more accurate when the foreground contained a consistent object than when it contained an inconsistent object. They argue that the data are consistent with a scene perception process in which object and scene recognition are highly interactive.

It is likely, therefore, that consistent objects in the present experiments are recognized relatively quickly. Inconsistent objects, in contrast, will be recognized more slowly, because the recognition of such objects is not supported by recognition of the scene itself. Indeed, the recognition of such objects might be substantially impaired when they are embedded in an inappropriate context and presented very briefly. This object recognition difficulty may constitute a region of perceptual dysfluency that draws attention (Johnston et al., 1990).

According to this account, attention is drawn to the inconsistent object because its identity is difficult to determine. Although Gordon (2004) argued for a semantic conflict account, in which attention is drawn to the location of conflict between an object and its context, some aspects of those data were consistent with the local processing difficulty explanation. For example, using the same scenes used in the present experiments, Gordon found that subjects were able to correctly identify inconsistent objects on fewer than $15 \%$ of trials. In contrast, the subjects were able to correctly identify consistent objects nearly $40 \%$ of the time. Although some of this difference likely reflects a response bias to choose consistent object options under conditions of uncertainty, it may also reflect genuine difficulty in perceiving the inconsistent object.

Henderson et al. (1999) argued that attention is guided within a scene by activation in a "saliency map." According to this account, objects that are most salient are likely to attract attention (and the eyes). Although Henderson et al. argued that selection during initial scene viewing is determined entirely on the basis of perceptual features (such as color, luminance, or texture), the present results suggest that prioritization of objects within a complex scene may also be influenced by the ease with which such objects are identified; objects whose identities are difficult to determine (such as inconsistent objects within meaningful scenes) receive high priority for selection.

As attention selects objects (on the basis of perceptual salience or local processing difficulty), representations of other objects within the scene appear to be suppressed. The consequences of this suppression on subsequent attention may be limited, given the saliency map framework described above. If all unselected objects are equally likely to be suppressed, then the relative priority of each object within the saliency map should remain the same. However, the suppression may affect the representations of those objects, and may manifest as negative priming when subjects are required to respond to the inhibited objects after scene presentation.

\section{Scene Perception and Priming}

The results of negative priming studies (e.g., Neill, 1977; Tipper, 1985, 2001) have been influential because of what they reveal about attention and about the representation of visual information. For example, Tipper's (1985) work demonstrated that selective attention to an object is accompanied by inhibition of other objects. Although the precise mechanism by which the inhibition operates remains a subject of controversy (see, e.g., Neill \& Valdes, 1996; Tipper, 2001), the argument that selective attention includes an inhibitory component is generally well-supported. In addition, Tipper (1985) demonstrated that semantic representations may be retrieved even for unattended objects, and that it is these representations upon which inhibition operates. Thus, the findings of such studies address both the mechanisms underlying selective attention as well as the representation of visual objects.

Because of this, the examination of priming from scene content appears to be a promising approach to the study of scene representation and attention. Results from such studies may reveal the attentional dynamics accompanying scene viewing, as they have in the present study. In addition, this approach can be used to explore the representation of objects in scenes. By manipulating the relationship between the scene elements and the target, one can further investigate the nature of such representations. For example, using word targets that are semantically related to scene objects may reveal the level at which inhibition operates, just as Tipper and Driver (1988) demonstrated that ignoring a picture of a cat can negatively 
prime $d o g$. Similarly, manipulating the physical similarity of the scene objects and target objects may further reveal the nature of object representations formed during scene viewing. Thus, the present experiments represent a new approach that may be applied to investigate a number of issues that are key to understanding the perception and representation of scenes.

Previous research has typically demonstrated negative priming in situations in which two or more objects are placed explicitly in competition for resources. For example, on each trial of Tipper and Driver's (1988) experiment, subjects viewed two overlapping objects. One of these objects was the target to be named, whereas the other was a distractor to be ignored. In contrast, in the present experiments, there was no task that explicitly placed objects in the scene in competition with each other. The subjects did not name the objects, or respond to the objects themselves in any way at all. Given this, it is perhaps surprising that negative priming occurred in the present experiments; without competition, why should inhibition be necessary during scene viewing?

Before addressing this issue, it is important to make a distinction between competition that results from task requirements and competition that is a natural aspect of perceptual processing. Although it is certainly true that the subjects in the present study were not required to respond to any object during scene viewing, there is considerable evidence that competition among object representations exists even in the absence of experimenter-imposed task demands. As VanRullen and Koch (2003) have argued, competition may occur naturally in any cluttered environment, regardless of an observer's goals. For example, Kastner, de Weerd, Desimone, and Ungerleider (1998) have shown evidence of competition between object representations in area V4; such competition occurred even when subjects were not required to respond to the competing stimuli. Thus, the fact that subjects in the present study were not explicitly required to respond to the objects embedded in the scenes does not imply that those objects do not compete for attention, or that inhibition is unlikely to occur.

Even if there were no competition among the objects in the scenes presented here, however, there may nonetheless be reason to expect suppression of consistent object representations. As others have argued (e.g., Houghton \& Tipper, 1994), inhibition may be an inherent aspect of selection, and this may be true regardless of externally imposed task demands. According to this view, selection is implemented through the action of two mechanisms: one that enhances the processing of the selection target, and another that suppresses the processing of other objects in the environment. Neurophysiological investigations generally support this view of attention (e.g., Desimone \& Duncan, 1995; Moran \& Desimone, 1985). This account offers one explanation for why consistent object representations should be suppressed in the present experiments, despite the fact that the task does not require it: If selection is routinely accompanied by inhibition, and if attention regularly selects inconsistent objects during a fixation, then inhibition of consistent objects is to be expected. The results of the present experiments suggest that this is indeed the case.

\section{Conclusions}

The present experiments demonstrate that attention is drawn to the locations of semantically inconsistent objects very early in scene viewing. Furthermore, the results suggest that inconsistent objects draw attention because they constitute areas of local perceptual processing difficulty. Together with the results of Gordon (2004), the present study provides converging evidence that attention is guided by the semantic processing of scenes and objects, and that selection of objects in scenes is accompanied by suppression of other objects within the scene. Selective attention therefore has profound consequences for how the scene is represented. These results provide important new insights into how we come to perceive and represent the visual information that surrounds us.

\section{REFERENCES}

Biederman, I. (1990). Higher-level vision. In D. N. Osherson, S. M. Kosslyn, \& J. M. Hollerbach (Eds.), Visual cognition and action: An invitation to cognitive science (Vol. 2, pp. 41-72). Cambridge, MA: MIT Press.

Biederman, I., Mezzanotte, R. J., \& Rabinowitz, J. C. (1982). Scene perception: Detecting and judging objects undergoing relational violations. Cognitive Psychology, 14, 143-177.

BoyCE, S. J., \& POLLATSEK, A. (1992). Identification of objects in scenes: The role of scene background in object naming. Journal of Experimental Psychology: Learning, Memory, \& Cognition, 18, 531-543.

DAVENPORT, J. L., \& POTTER, M. C. (2004). Scene consistency in object and background perception. Psychological Science, 15, 559-564.

De Graef, P., Christiaens, D., \& D’Ydewalle, G. (1990). Perceptual effects of scene context on object identification. Psychological Research, 52, 317-329.

DE Graef, P., De Troy, A., \& D’Y Dewalle, G. (1992). Local and global contextual constraints on the identification of objects in scenes. Canadian Journal of Psychology, 46, 489-508.

DESIMONE, R., \& DunCAN, J. (1995). Neural mechanisms of selective visual attention. Annual Review of Neuroscience, 18, 193-222.

Fox, E. (1995). Negative priming from ignored distractors in visual selection: A review. Psychonomic Bulletin \& Review, 2, 145-173.

FranCIS, W. N., \& KuČERA, H. (1982). Frequency analysis of English usage: Lexicon and grammar. Boston: Houghton Mifflin.

Gordon, R. D. (2004). Attentional allocation during the perception of scenes. Journal of Experimental Psychology: Human Perception \& Performance, 30, 760-777.

GRIMES, J. (1996). On the failure to detect changes in scenes across saccades. In K. A. Akins (Ed.), Perception (Vancouver Studies in Cognitive Science, Vol. 5, pp. 89-110). London: Oxford University Press.

Henderson, J. M., Weeks, P. A., \& Hollingworth, A. (1999). The effects of semantic consistency on eye movements during complex scene viewing. Journal of Experimental Psychology: Human Perception \& Performance, 25, 210-228.

HOLLINGWORTH, A. (2003). Failures of retrieval and comparison constrain change detection in natural scenes. Journal of Experimental Psychology: Human Perception \& Performance, 29, 388-403.

HOLLINGWORTH, A. (2004). Constructing visual representations of natural scenes: The roles of short- and long-term visual memory. Journal of Experimental Psychology: Human Perception \& Performance, 30, 519-537.

HOLLINGWORTH, A. (2005). The relationship between online visual representation of a scene and long-term scene memory. Journal of Experimental Psychology: Learning, Memory, \& Cognition, 31, 396-411.

HollingWORTH, A., \& Henderson, J. M. (1998). Does consistent scene context facilitate object perception? Journal of Experimental Psychology: General, 127, 398-415. 
Hollingworth, A., \& Henderson, J. M. (1999). Object identification is isolated from scene semantic constraint: Evidence from object type and token discrimination. Acta Psychologica, 102, 319-343.

Hollingworth, A., \& Henderson, J. M. (2002). Accurate visual memory for previously attended objects in natural scenes. Journal of Experimental Psychology: Human Perception \& Performance, 28, 113-136.

HOUGHTON, G., \& TiPPER, S. P. (1994). A model of inhibitory mechanisms in selective attention. In D. Dagenbach \& T. H. Carr (Eds.), Inhibitory processes in attention, memory, and language (pp. 53-112). San Diego: Academic Press.

IRWIN, D. E., \& ANDREWS, R. V. (1996). Integration and accumulation of information across saccadic eye movements. In T. Inui \& J. L. McClelland (Eds.), Attention and performance XVI: Information integration in perception and communication (pp. 125-155). Cambridge, MA: MIT Press, Bradford Books.

Irwin, D. E., \& Gordon, R. D. (1998). Eye movements, attention and trans-saccadic memory. Visual Cognition, 5, 127-155.

IRWIN, D. E., \& Zelinsky, G. J. (2002). Eye movements and scene perception: Memory for things observed. Perception \& Psychophysics, 64, 882-895.

Johnston, W. A., Hawley, K. J., Plewe, S. H., Elliott, J. M. G., \& DeWitT, M. J. (1990). Attention capture by novel stimuli. Journal of Experimental Psychology: General, 119, 397-411.

Kastner, S., De Weerd, P., Desimone, R., \& Ungerleider, L. G. (1998). Mechanisms of directed attention in the human extrastriate cortex as revealed by functional MRI. Science, 282, 108-111.

Loftus, G. R., Hanna, A. M., \& LesteR, L. (1988). Conceptual masking: How one picture captures attention from another picture. Cognitive Psychology, 20, 237-282.

LoFTUS, G. R., \& MACKWORTH, N. H. (1978). Cognitive determinants of fixation location during picture viewing. Journal of Experimental Psychology: Human Perception \& Performance, 4, 565-572.

MCCONKIE, G. W., \& ZoLA, D. (1979). Is visual information integrated across successive fixations in reading? Perception \& Psychophysics, 25, 221-224.

MitrofF, S. R., Simons, D. J., \& Levin, D. T. (2004). Nothing compares 2 views: Change blindness can occur despite preserved access to the changed information. Perception \& Psychophysics, 66, 1268-1281.

Moran, J., \& Desimone, R. (1985). Selective attention gates visual processing in the extrastriate cortex. Science, 229, 782-784.

NeELY, J. H. (1991). Semantic priming effects in visual word recognition: A selective review of current findings and theories. In D. Besner \& G. W. Humphreys (Eds.), Basic processes in reading: Visual word recognition (pp. 264-336). Hillsdale, NJ: Erlbaum.

NeILL, W. T. (1977). Inhibitory and facilitatory processes in selective attention. Journal of Experimental Psychology: Human Perception \& Performance, 3, 444-450.

NeILL, W. T., \& VALDES, L. A. (1996). Facilitatory and inhibitory aspects of attention. In A. F. Kramer, M. G. H. Coles, \& G. D. Logan (Eds.), Converging operations in the study of visual selective attention (pp. 77-106). Washington, DC: American Psychological Association.
O’Regan, J. K., Deubel, H., Clark, J. J., \& Rensink, R. A. (2000). Picture changes during blinks: Looking without seeing and seeing without looking. Visual Cognition, 7, 191-211.

O'Regan, J. K., Rensink, R. A., \& Clark, J. J. (1999). Changeblindness as a result of "mudsplashes." Nature, 398, 34.

Ortells, J. J., \& Tudela, P. (1996). Positive and negative semantic priming of attended and unattended parafoveal words in a lexical decision task. Acta Psychologica, 94, 209-226.

Potter, M. C. (1976). Short-term conceptual memory for pictures. Journal of Experimental Psychology: Human Learning \& Memory, 2,509-522.

RENSINK, R. A. (2000). The dynamic representation of scenes. Visual Cognition, 7, 17-42.

RENSINK, R. A., O'REGAN, J. K., \& CLARK, J. J. (1997). To see or not to see: The need for attention to perceive changes in scenes. Psychological Science, 8, 368-373.

SchVANEVElDT, R. W., \& Meyer, D. E. (1973). Retrieval and comparison processes in semantic memory. In S. Kornblum (Ed.), Attention and performance IV (pp. 395-409). New York: Academic Press.

Schyns, P. G., \& Oliva, A. (1994). From blobs to boundary edges: Evidence for time- and spatial-scale-dependent scene recognition. Psychological Science, 5, 195-200.

SERENO, J. A. (1991). Graphemic, associative, and syntactic priming effects at a brief stimulus onset asynchrony in lexical decision and naming. Journal of Experimental Psychology: Learning, Memory, \& Cognition, 17, 459-477.

TIPPER, S. P. (1985). The negative priming effect: Inhibitory priming by ignored objects. Quarterly Journal of Experimental Psychology, 37A, 571-590.

TIPPER, S. P. (2001). Does negative priming reflect inhibitory mechanisms? A review and integration of conflicting views. Quarterly Journal of Experimental Psychology, 54A, 321-343.

TipPeR, S. P., Brehaut, J. C., \& Driver, J. (1990). Selection of moving and static objects for the control of spatially directed action. Journal of Experimental Psychology: Human Perception \& Performance, 16, 492-504.

TiPPER, S. P., \& CRANSTON, M. (1985). Selective attention and priming: Inhibitory and facilitatory effects of ignored primes. Quarterly Journal of Experimental Psychology, 37A, 591-611.

TIPPER, S. P., \& DrIVER, J. (1988). Negative priming between pictures and words in a selective attention task: Evidence for semantic processing of ignored stimuli. Memory \& Cognition, 16, 64-70.

VANDERWART, M. (1984). Priming by pictures in lexical decision. Journal of Verbal Learning \& Verbal Behavior, 23, 67-83.

VANRullen, R., \& KoCH, C. (2003). Competition and selection during visual processing of natural scenes and objects. Journal of Vision, $\mathbf{3}$, 75-85.

VoLKMANN, F. C. (1986). Human visual suppression. Vision Research, 26, 1401-1416.

(Manuscript received January 25, 2005; revision accepted for publication July 26, 2005.) 